\title{
How can we apply the use of surface- enhanced Raman scattering nanoparticles in tumor imaging?
}

\author{
"The high precision in visualizing the true extent of \\ tumor spread afforded by the newest generations of \\ SERRS nanoparticles will increase the accuracy with which \\ cancer can be diagnosed, resected or destroyed..."
}

First draft submitted: 2 November 2016; Accepted for publication: 10 November 2016; Published online: 12 January 2017

Keywords: cancer • imaging • nanoparticles $\bullet$ Raman $\bullet$ SERRS $\bullet$ SERS $\bullet$ tumor

\section{Background on the Raman effect}

When light interacts with matter, most photons are scattered elastically via the Rayleigh effect. A small fraction of photons, however ( $\sim 1$ out of 10 million), undergoes a different scattering mechanism, where an energy exchange occurs during the interaction of the incident photon with the scattering material [1]. This phenomenon was discovered in 1928 by Chandrasekhara Venkata Raman [2], for which he received the Nobel Prize in 1930. Why is the Raman effect so important? Because the energy exchange during the interaction of Raman photons and matter is specific to the molecular composition of the scattering material. By measuring the change in wavelength that Raman photons experience, one can deduce the molecular composition of the scattering material from the resulting spectrum (Raman 'fingerprint'), and this principle has opened up many different applications [3].

A major limitation of intrinsic Raman spectroscopy is that the fraction of inelastically scattered Raman photons compared with the elastically scattered Rayleigh photons is very small, requiring long acquisition times, which surpass what would be feasible in most biomedical settings, especially in human patients. In 1973, Fleischmann et al. conducted the first experiments that led to the discovery of what would later be called surface-enhanced Raman scattering (SERS), a method that allows major enhancements in the intensity of the Raman signal [4]. The SERS enhancement is believed to be due to a combination of several contributing phenomena [5]. The one most frequently cited factor is surface plasmon resonance caused by collective oscillations of electrons in the conduction band of, for example, gold, silver or copper nanoparticle surfaces, with other factors being detailed elsewhere [5].

\section{SERS nanoparticles}

For medical applications, the next logical step was to integrate the SERS amplification principle into nanoparticles with the goal of in vitro sensing, and further to develop contrast agents for in vivo use. In order to bridge the gap from in vitro or ex vivo use to in vivo use with true translational potential, multiple additional factors had to be considered and hurdles had to be overcome.

- First of all, the materials used have to be considered. From the three materials best known to create high SERS enhancement, that is to say, gold, silver and copper, gold has the advantage of being the most inert material and thus having the highest chance for clinical translation;

- The nanoparticle synthesis method plays an important role, as it determines, for
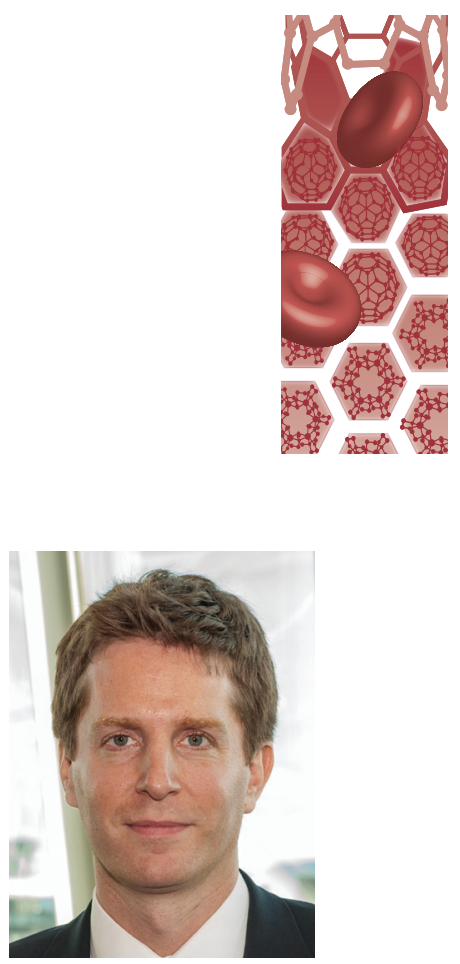

Moritz F Kircher

Department of Radiology, Memorial

Sloan Kettering Cancer Center,

1275 York Avenue, New York, NY 10065, USA

and

Center for Molecular Imaging \&

Nanotechnology (CMINT), Memorial

Sloan Kettering Cancer Center,

1275 York Avenue, New York, NY 10065, USA

and

Weill Cornell Medical College of Cornell University, 1300 York Avenue, New York, NY 10065, USA

kircherm@mskcc.org
Future $\because$ Medicine part of 
example, if potentially toxic surfactants are used and incorporated in the nanoparticle;

- Nanoparticles for in vivo use have to be shielded in a way that the Raman reporter is encapsulated and isolated from external stimuli in order to preserve the unique identifying Raman fingerprint that would allow its detection.

Some of the first studies, to demonstrate that SERS imaging could be performed in living subjects, used a nanoparticle preparation from the company Oxonica Materials, Inc. (CA, USA; now Cabot Corporation, GA, USA). In these initial studies, spherical gold core/ silica shell nanoparticles were injected into healthy mice intravenously and their accumulation in the liver was recorded [6].

\section{In vivo tumor imaging with SERS nanoparticles}

Subsequently, one of the first examples of in vivo imaging of tumors was reported in 2012, which made use of a gold core/silica shell/Gd-coated nanoparticle that allowed triple modality imaging of brain tumors using SERS, MRI and photoacoustic imaging [7]. This nanoparticle construct did not use a targeting moiety on its surface, relying on the enhanced permeability and retention (EPR) effect for passive accumulation within glioblastoma tumors. The detection threshold of this probe was approximately $600 \mathrm{fM}$, as measured in a tissue-mimicking agarose phantom. It allowed precise detection of the main tumor and microscopic extensions, and the feasibility of in vivo tumor resections based on the SERS signal was demonstrated for the first time [7]. Importantly, it was also shown that no nanoparticles accumulated in the healthy brain, an observation establishing targeting specificity. However, when my group continued this work and tested the same nanoparticles in other extracranial tumor models, the resultant SERS signal was not found to be sufficient to allow robust cancer imaging.

"We therefore sought to systematically investigate and optimize the multitude of factors that contribute to the SERS enhancement."

Based on these observations, we concluded that the EPR effect for these particles is higher in the glioblastoma mouse models (U87MG and TS543 [7], RCAS/ tv-a [8]) than in the extracranial tumor models. We hypothesized that if SERS nanoparticles could be developed with markedly higher signal intensity than those previous generations from Oxonica/Cabot, we could overcome this hurdle by surpassing the needed detection threshold, and make other, ideally all, tumor types visible. After all, the existence of the EPR effect in virtually all tumor types had been described extensively by Maeda et al. [9]. It might just not have been possible to create a universal tumor imaging agent based on the EPR effect alone before, because previously tried imaging modalities did not have the necessary sensitivity to do so.

\section{Resonant SERS nanoparticles for universal tumor imaging}

We therefore sought to systematically investigate and optimize the multitude of factors that contribute to the SERS enhancement. This included the size, geometry and surface chemical composition of the gold core; the chemistry of the Raman reporter; and the silication method. These investigations revealed several areas where major improvements were possible, such as a new primer-free silication method that would preserve more Raman reporter molecules on the gold surface. The resulting resonant SERS (SERRS) nanoparticles achieved a detection threshold in the low femtomolar or even attomolar range $[10,11]$, and thus resulted in a detection sensitivity up to 6000 -fold higher than their Oxonica/Cabot counterparts.

Indeed, without requiring any targeting moiety, these new generations of SERRS nanoparticles were able to visualize tumors in many different cancer types. We tested the same nanoparticle (SERRS nanostar) in mouse models that closely recapitulate human breast, prostate, brain, liver, sarcoma and pancreatic cancer [11-13]. We conclude that it is the vastly increased signal enhancement factors that enabled overcoming the sensitivity threshold that previously had not yet been reached. Of course, there may be other important factors, such as size, geometry and surface charge/ composition, that likely play a role in how efficiently the nanoparticles home to tumors and thus contribute to the overall intratumoral signal achieved.

\section{Actively targeted SERS nanoparticles for tumor imaging}

An important question and active topic of debate in the nanotechnology community is the role of active versus passive targeting. In our experience so far, employing ultrasensitive, passively targeted SERRS nanoparticles has enabled excellent visualization of not only the bulk tumors, but also microscopic extensions, and even microscopic locoregional satellite metastases in multiple genetically engineered mouse models. However, we have observed in direct intraindividual comparisons that the addition of an active targeting moiety can help visualize some unexpected locations of tumor spread that the nontargeted particles 'miss' [14,15]. This is most likely due to the fact that the ligand on SERS nanoparticles can bind to endoluminally expressed receptors, whereas nontargeted SERS nanoprobes are unable to 
arrest in the tumor microenvironment if the integrity of the capillary bed is not compromised.

\section{SERS multiplexing}

Besides the very high sensitivity and specificity of the Raman signal via the spectroscopic fingerprint, SERS nanoparticles have a third major advantage: multiplexing. Modifications of the chemical structure of the Raman reporter can suffice to create many nanoparticles that have the same size and surface composition, but are spectrally distinct from each other. This could, in theory, enable the depiction of many different markers of interest $(>10)$ in the same tumor by using the SERS nanoparticles as tags for tumor marker expression profiling. The main challenges that would need to be overcome toward such a goal is the synthesis of Raman reporters that are spectrally unique, result in similar signal intensities and can be incorporated into otherwise identical nanoparticles. This can represent a significant challenge at the synthetic level.

\section{Future perspective}

While SERS nanoparticles, especially using multiplexing, could serve as a new method to image cancer spread and intratumoral heterogeneity in preclinical models, the ultimate goal is clinical translation.

One obstacle toward application in human tumor surgeries is that clinical Raman scanners with the needed wide field of view and rapid image acquisition speeds have not yet been developed. However, progress is being made toward this end [16]. Another area of use in oncology is the endoscopic detection of tumors, and for this purpose the needed hardware, in other words, a Raman endoscope that fits into the instrument channel of a conventional white light endoscope, already exists [17]. A highly promising field is the development of deep tissue Raman detectors, which are based on the surface-enhanced 'spatially offset' Raman scattering principle. Surface-enhanced spatially offset Raman scattering detectors were shown to enable detection of Raman signals through several centimeters of tissue [18].

The real bottleneck of clinical SERS cancer imaging will not be the detection technology, but the regulatory approval of the nanoparticles themselves. To this

\section{References}

1 Andreou C, Kishore SA, Kircher MF. Surface-enhanced Raman spectroscopy: a new modality for cancer imaging. J. Nucl. Med. 56(9), 1295-1299 (2015).

2 Raman CV, Krishnan KS. A new type of secondary radiation. Nature 121(3048), 501-502 (1928).

3 Zavaleta CL, Kircher MF, Gambhir SS. Raman's 'effect' on molecular imaging. J. Nucl. Med. 52, 1839-1844 (2011). end, it is promising that SERS nanoparticles consisting of inert materials, such as gold and silica, have shown a favorable toxicity profile in extensive animal studies [19], and other types of gold and gold-silica nanoparticles for cancer therapy have already advanced into clinical trials [20].

Thus, despite some hurdles that still need to be overcome, SERS nanoparticles should have a realistic chance for clinical translation in the near future. The high precision in visualizing the true extent of tumor spread afforded by the newest generations of SERRS nanoparticles will increase the accuracy with which cancer can be diagnosed, resected or destroyed, thus harboring tremendous promise. Increased accuracy means that less tumor tissue is left behind and more healthy tissue can be spared. This could improve patient outcomes not only during classical open surgeries, but also especially in the emerging fields of minimally invasive and robotically assisted procedures.

\section{Financial \& competing interests disclosure}

The author gratefully acknowledges funding from the $\mathrm{NIH}$ (EB017748 and CA16396), the Damon Runyon-Rachleff Innovation Award (DRR-29-14), the Pershing Square Sohn Prize and an MSKCC Technology Development Grant. Acknowledgements are also extended to the grant-funding support provided by the MSKCC NIH Core Grant (P30-CA008748). The author is an inventor of several pending patents related to the topic of this article, and a cofounder of RIO Imaging, Inc., a startup company that seeks to translate Raman nanoparticle technology into the clinics. The author has no other relevant affiliations or financial involvement with any organization or entity with a financial interest in or financial conflict with the subject matter or materials discussed in the manuscript apart from those disclosed.

No writing assistance was utilized in the production of this manuscript.

\section{Open access}

This work is licensed under the Attribution-NonCommercialNoDerivatives 4.0 Unported License. To view a copy of this license, visit http://creativecommons.org/licenses/by-ncnd/4.0/

4 Fleischmann M, Hendra PJ, Mcquillan AJ. Raman-spectra of pyridine adsorbed at a silver electrode. Chem. Phys. Lett. 26(2), 163-166 (1974).

5 Lombardi JR, Birke RL. The theory of surface-enhanced Raman scattering. J. Chem. Phys. 136(14), 144704 (2012).

6 Keren S, Zavaleta C, Cheng Z, De La Zerda A, Gheysens O, Gambhir SS. Noninvasive molecular imaging of small living subjects using Raman spectroscopy. Proc. Natl Acad. Sci. USA 105, 5844-5849 (2008). 
7 Kircher MF, De La Zerda A, Jokerst JV et al. A brain tumor molecular imaging strategy using a new triple-modality MRI-photoacoustic-Raman nanoparticle. Nat. Med. 18, 829-834 (2012).

8 Karabeber H, Huang R, Iacono P et al. Guiding brain tumor resection using surface-enhanced Raman scattering nanoparticles and a hand-held Raman scanner. ACS Nano 8(10), 9755-9766 (2014).

9 Maeda H, Wu J, Sawa T, Matsumura Y, Hori K. Tumor vascular permeability and the EPR effect in macromolecular therapeutics: a review. J. Control Release 65(1-2), 271-284 (2000).

10 Harmsen S, Bedics MA, Wall MA, Huang R, Detty MR, Kircher MF. Rational design of a chalcogenopyrylium-based surface-enhanced resonance Raman scattering nanoprobe with attomolar sensitivity. Nat. Commun. 6, 6570 (2015).

11 Harmsen S, Huang R, Wall MA et al. Surfaceenhanced resonance Raman scattering nanostars for highprecision cancer imaging. Sci. Transl. Med. 7(271), 271ra7 (2015).

12 Andreou C, Neuschmelting V, Tschaharganeh DF et al. Imaging of liver tumors using surface-enhanced Raman scattering nanoparticles. ACS Nano 10(5), 5015-5026 (2016).

13 Spaliviero M, Harmsen S, Huang R et al. Detection of lymph node metastases with SERRS nanoparticles. Mol. Imaging Biol. 18(5), 677-685 (2016).
14 Huang R, Harmsen S, Samii JM et al. High precision imaging of microscopic spread of glioblastoma with a targeted ultrasensitive SERRS molecular imaging probe. Theranostics 6(8), 1075-1084 (2016).

15 Oseledchyk A, Andreou C, Wall MA, Kircher MF. Folatetargeted surface-enhanced resonance Raman scattering nanoprobe ratiometry for detection of microscopic ovarian cancer. ACS Nano.doi:10.1021/acsnano.6b0679 (2017) (Epub ahead of print).

16 Bohndiek SE, Wagadarikar A, Zavaleta CL et al. A small animal Raman instrument for rapid, wide-area, spectroscopic imaging. Proc. Natl Acad. Sci. USA 110(30), 12408-12413 (2013).

17 Zavaleta CL, Garai E, Liu JT et al. A Ramanbased endoscopic strategy for multiplexed molecular imaging. Proc. Natl Acad. Sci. USA 110 (25), E2288-E2297 (2013).

18 Stone N, Kerssens M, Lloyd GR, Faulds K, Graham D, Matousek P. Surface enhanced spatially offset Raman spectroscopic (SESORS) imaging - the next dimension. Chem. Sci. 2(4), 776 (2011).

19 Thakor AS, Luong R, Paulmurugan R et al. The fate and toxicity of Raman-active silica-gold nanoparticles in mice. Sci. Transl. Med. 3(79), 79ra33 (2011).

20 Thakor AS, Gambhir SS. Nanooncology: the future of cancer diagnosis and therapy. CA Cancer J. Clin. 63(6), 395-418 (2013). 